\title{
Intercalation of a Cationic Cyanine Dye Assisted by Anionic Surfactants within Mg-Al Layered Double Hydroxide
}

\author{
Rina Sato, Shingo Machida, Minoru Sohmiya, Yoshiyuki Sugahara, and Régis Guégan*
}

Cite This: ACS Omega 2021, 6, 23837-23845

Read Online

ABSTRACT: An original route for the intercalation of a $1,1^{\prime}$ diethyl-2,2'-cyanine iodide (PIC) cationic dye, through the use of anionic surfactants as vector/carrier phases, within $\mathrm{Mg}-\mathrm{Al}$ layered double hydroxide $(\mathrm{LDH})$ was investigated. From the data acquired from complementary techniques (X-ray diffraction, infrared and UV-visible spectroscopies, thermogravimetry, and fluorimetry), it appears that both the intercalation and aggregation states of the cationic dye within the internal structure of LDH mainly depend on both the surfactant state (monomer form or spherical micelle) and its amount. The intercalation of PIC at a low molar ratio to the anionic surfactant leads to the formation of $J$-aggregates with singular fluorescence properties that mainly depend on the nature of the anionic surfactant used for the co-intercalation process. The results obtained in this study open new routes for the intercalation of cationic species, assisted by anionic surfactants, within LDHs.

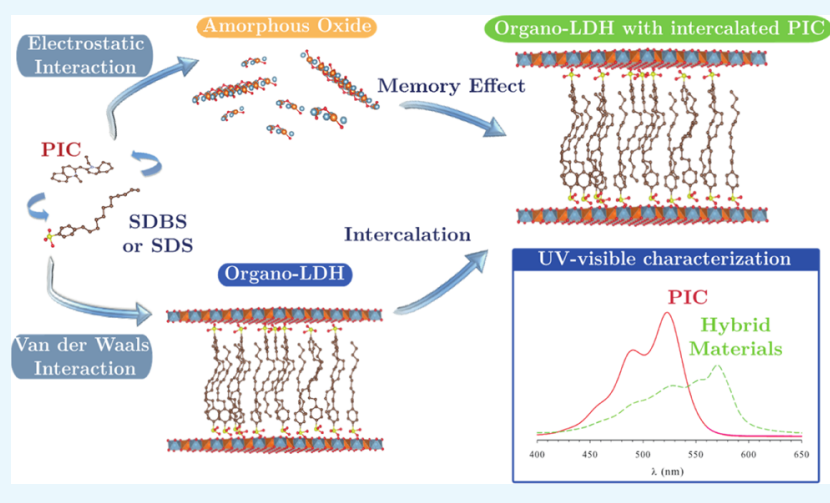

\section{INTRODUCTION}

Layered materials such as layered double hydroxides (LDHs) and clay minerals have been commonly used as confinement matrixes for organic photoactive species (dye). ${ }^{1,2}$ In addition to prevent their possible degradation, the use of the $2 \mathrm{D}$ inorganic materials improves the organization of the dyes and their photoluminescent properties. ${ }^{3-8}$ Indeed, in contrast to bulk solid dyes where multiple organizations can be formed, showing a lack of consistence in the photoluminescent properties with possible red-shifted fluorescence, line broadening, and even luminescence quenching, the intercalation of dyes within the interlayer space of the host inorganic matrixes drives to the formation of $J$-aggregates at very low dye contents. $^{4,9-12}$ In those singular aggregates with head-to-tail arrangement of the dye molecules that can be observed at large concentrations of bulk dye, the absorption occurs at a longer wavelength than that of the initial absorption band, with the observation of a sharp absorption band and resonance fluorescence. ${ }^{4-15}$

The confinement of organic compounds within layered materials bears on the insertion reaction, with the substitution of the inorganic ions, located within the interlayer space. ${ }^{3,16-26}$ For oxides derivatives from $\mathrm{LDHs}$, the reconstruction of the layered structure of materials occurs in the presence of anions in a solution. ${ }^{3,17,27}$ With these different possible routes, anionic dyes such as acid red-27, coumarin-3-carboxylate, perylene chromophores, and rhodamine B chromophores were intercalated through ion exchange into the $\mathrm{LDH}$ gallery. ${ }^{6,8,11,26}$ However, in these $\mathrm{LDH}$-dye hybrid materials, undesirable aggregates could still be formed, leading to poor luminescent properties. 7 Anionic surfactants, associated to dyes, are recognized to favor the formation of $J$-aggregates at lower concentrations than bulk dye solutions. In addition, if they were used a co-intercalated guests, a control of the orientation of the dye in LDH-based hybrid materials could be expected. In addition, the presence of a surfactant improves the luminescent performances by providing a nonpolar homogeneous environment. ${ }^{11}$

If various anionic dyes are successfully co-intercalated with anionic surfactants within the internal structure of LDHs, the intercalation is possible with respect to the opposite electric properties of host and two guest species. However, no studies tackle the possibility to associate cationic dyes such as cyanine ones with $\mathrm{LDH}$ through the use of anionic surfactants as vector/carrier phases. As exemplary of the original way for the insertion of confined species in layered materials, the successful intercalation of an anionic anti-inflammatory drug was assisted by a cationic surfactant in an organoclay. While showing a similar charge as that of the phyllosilicate sheets, the anionic

Received: May 25, 2021

Accepted: July 30, 2021

Published: September 10, 2021 


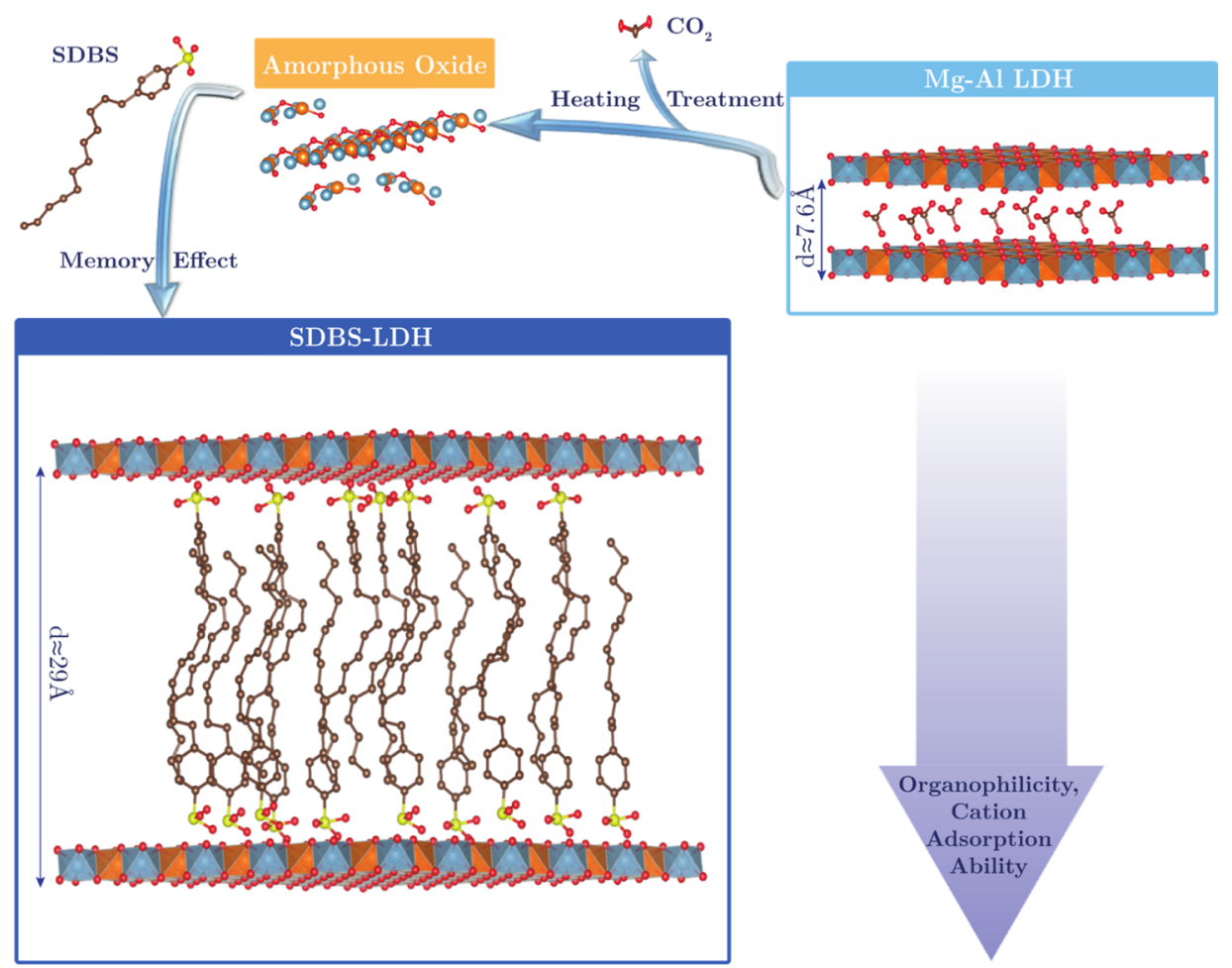

Figure 1. Schematic representation of the layered materials used in this study: $\mathrm{Mg}-\mathrm{Al} \mathrm{LDH}$ and SDBS-LDH (organo-LDH) as well as their preparation. Organo-LDH can be obtained through the oxidation of the initial $\mathrm{Mg}-\mathrm{Al} \mathrm{LDH}$ including the anionic surfactants in solution, allowing the reformation of a layered structure via a memory-reconstructive property. The resulting layered materials display a hydrophobic behavior with a large expansion of an interlayer space, as well as an ability to adsorb cationic species.

drug could be intercalated in a large content in the organoclay, expanding its interlayer galleries. ${ }^{20-22}$

A 1,1'-diethyl-2,2'-cyanine iodide (PIC) cationic dye was intensively used and associated with clay minerals. ${ }^{4,5,28}$ This cationic dye easily forms two-dimensional $\mathrm{H}$ - or $J$-aggregates with parallel organization of the stacked molecules showing slip angles. ${ }^{29}$ The delocalization of $\pi$ electrons in J-aggregates leads to resonant fluorescence that can find some echo in photographic processes and nonlinear optical applications, for instance. ${ }^{15}$ PIC cyanine dyes show an outstanding high extinction coefficient and easily form aggregates of which size is estimated to be from a couple to thousands of nanometers. PIC aggregates that have reached at a high dye concentration or with the use of electrolytes show, unlike other systems, high quantum yield for fluorescence with a small Stokes shift.

The different states of the adsorbed dyes onto layered materials are controlled not only by the characteristics of the inorganic layers such as the layer charge density, ${ }^{13,28}$ chemical composition, ${ }^{17,30}$ isomorphous substitution, and the particle size but also the nature and the amounts of surfactants that are intercalated. ${ }^{27,31}$ LDHs can be prepared by different routes of synthesis (co-precipitation and urea method), allowing a fine control of a chemical composition, charge density, and size of the particle and thus driving to versatile layered materials. ${ }^{27}$ The combination of J-aggregates formed by PIC with $\mathrm{LDH}$ may therefore lead to novel effects to be observed in organic dye onto the surface of layered materials.

The purposes of this study are (i) to use anionic surfactants as vector/carrier phases for the intercalation of PIC at high concentration within $\mathrm{LDH}$; (ii) to understand the role of the surfactant state below and above the critical micelle concentration $(\mathrm{cmc})$ and its nature on the formation of $J$ - aggregates; and (iii) to optimize the photoluminescent properties by modulating the orientation of the adsorbed dye by the density of the surfactant.

\section{RESULTS AND DISCUSSION}

If the intercalation of anionic dyes in LDHs reveals to be an easy operation, based on the substitution of the inorganic anions and/or the reconstruction of $\mathrm{LDH}$ derivative oxides in the layered structure in the presence of organic anions, the adsorption of cationic species or even nonionic species remains difficult. $^{3,6-8,13,16,27}$ However, recent studies have shown that for clay minerals, layered materials equivalent to $\mathrm{LDHs}$ showing an oppositive electric nature, an ability to also adsorb organic anions through the use of long-alkyl-chain cationic surfactants. Leaning against this strategy, an anionic antiinflammatory drug, diclofenac, defined as a persistent pollutant in water resources, could be co-intercalated with cationic surfactants within a clay mineral at very large concentrations, a process not reachable with the single use of raw minerals as adsorbents. $^{20-23}$

Thus, using an equivalent experimental protocol based on the use of surfactants as vector/carrier phases for adsorption, one of the objectives of this study is to extend this procedure to LDH with the use of anionic surfactants and validate it with a cationic dye recognized for old-type J-aggregates at high concentrations. ${ }^{15,29,32}$

3.1. Characteristics of the Layered Materials. On the basis of both X-ray diffraction (XRD) with, in particular, the observation of a large shift at a low angle value of the (003) reflection probing the proper intercalation of the anionic surfactants and Fourier transform infrared (FTIR) data focusing on the $\mathrm{CH}_{2}$ stretching vibration (Figures S1-S7), 

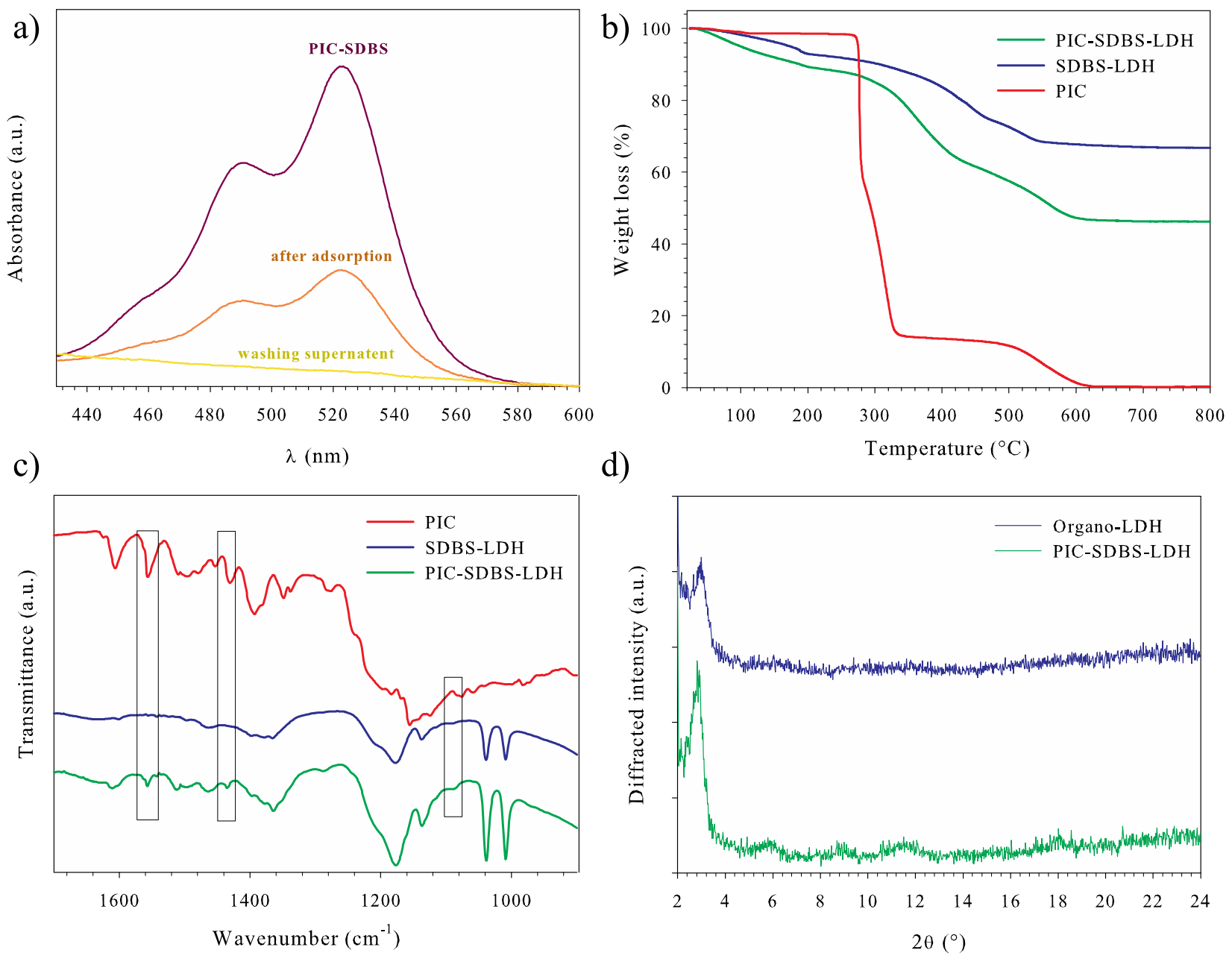

d)

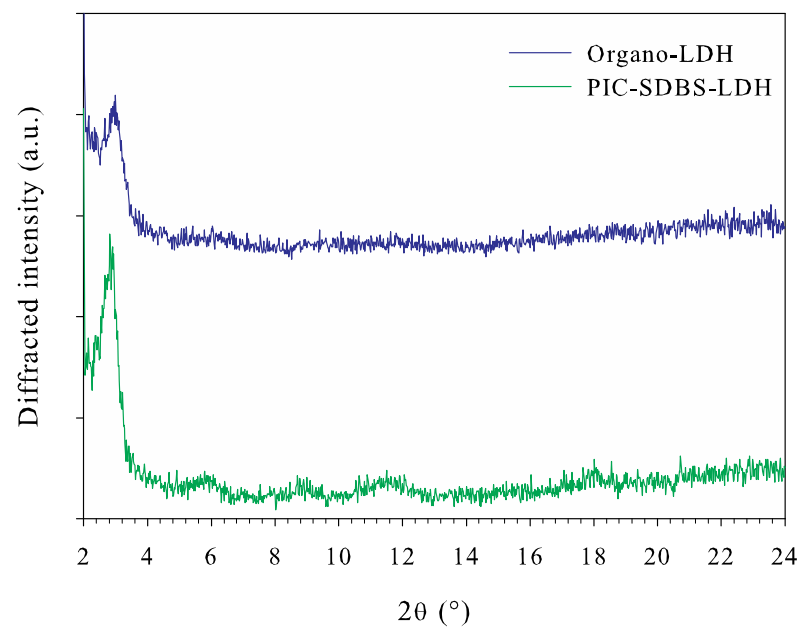

Figure 2. (a) UV-vis absorption spectra of the PIC-SDBS solutions before and after being mixed with LDHs in purple and orange colors, respectively. The supernatant (yellow) collected after a washing procedure of the PIC-SDBS-LDH samples did not exhibit any release of PIC. (b) TG analyses of PIC (red), SDBS-LDH (blue), and PIC-SDBS-LDH (green). (c) FTIR spectra of PIC (red), SDBS-LDH (blue), and PIC-SDBSLDH (green). (d) XRD patterns of organo-LDH (blue) and PIC-SDBS-LDH (green).

sodium dodecylbenzenesulfonate (SDBS) is organized in a monolayer arrangement within an interlayer space which is expanded at a value of about $2.9 \mathrm{~nm}$, a result consistent to other studies (SDBS-LDH). ${ }^{16,33,34} \mathrm{Mg}-\mathrm{Al} \mathrm{LDH}$ and its organically modified derivatives display versatile and complementary properties: an anion exchange capacity, hydrophobicity, and large expansion of the interlayer space, facilitating the possible intercalation or adsorption of organic species. ${ }^{11}$ In addition, the prior intercalation of the SDBS anionic surfactant provides a slight excess of negative charges to the hybrid materials, allowing one to expect the further adsorption of cationic compounds (Figure 1). 6,11,16

3.2. Intercalation of PIC into the Layered Materials. To confirm the possible intercalation of the PIC within the internal structure of the layered materials, the experiments were carried out on a relatively high concentration range $(7.05$ $\times 10^{-3}$ and $6.39 \times 10^{-2} \mathrm{~mol} \mathrm{~L}^{-1}$ for PIC and SDBS, respectively, at which the surfactant forms $L_{1}$ spherical micelles) to facilitate the analysis and the identification of the signals related to the PIC by both UV-vis and infrared spectroscopies. Indeed, if the amount of PIC is rather small, it is difficult to probe their presence by XRD or FTIR except through fluorescence spectroscopic analyses as it will be discussed later. Thus, it is expected that the following study at a high concentration of PIC and its location within the layered materials may be transposed to the low concentration regime of a dye for the preparation of the hybrid materials. In addition, further information related to the location of the dye can be obtained with the use of XRD by following any changes of the $00 l$ reflections if the loading of the organic species is sufficient. The latter (i.e., adsorbed or intercalated amount of PIC) can be probed by thermogravimetry (TG) analyses of the solid hybrid materials that can be corroborated by UV spectroscopy with the analyses of the PIC solution before and after being in contact with the (organo)layered materials or/and the derivative oxides.

The UV-vis absorption spectra of a solution containing both PIC-SDBS before and after adsorption onto the organo$\mathrm{LDH}$ (Figure $2 \mathrm{a}$ ) reveal the absorption band characteristics of a cationic dye at 440-550 nm. 4,15,29,32 The maximum of the absorption is observed at $525 \mathrm{~nm}$, with additional satellite bands at shorter wavelengths ( 488 and $460 \mathrm{~nm}$ ). The presence of the anionic surfactants slightly shifts the maximum to larger wavelengths (red shift), the monomer band of PIC, expected to be observed for a bulk dilute aqueous PIC solution at 521 $\mathrm{nm}$. At a concentration of $6.39 \times 10^{-2} \mathrm{~mol} \mathrm{~L}^{-1}$ above the $\mathrm{cmc}$, SDBS forms spherical micelles ( $\mathrm{L}_{1}$ phase) that affect the environment of PIC with the possible trapping within the core 


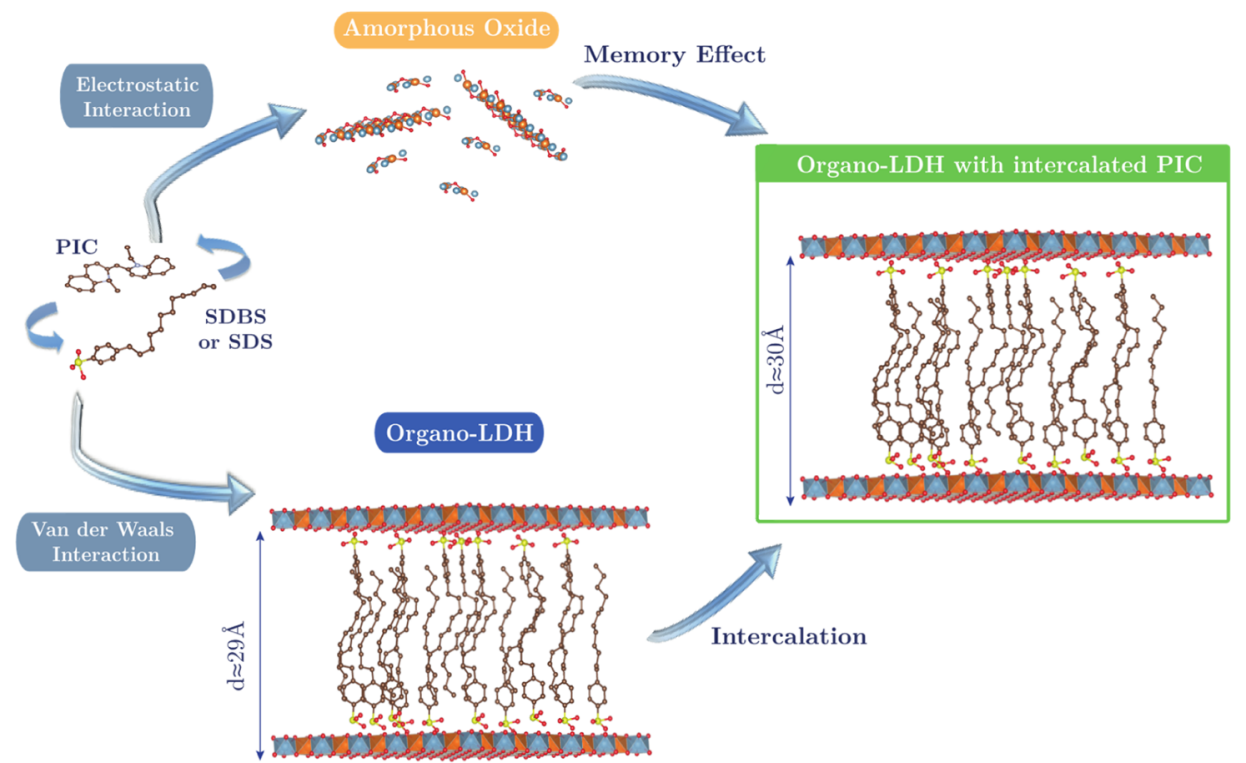

Figure 3. Schematic representation of the different routes and interactional mechanisms for the intercalation of a cationic dye (PIC) within the interlayer space of LDHs assisted by the use of anionic surfactants (SDBS or SDS). The formation of organic complexes strengthened by electrostatic interaction below or above the critical concentration $(\mathrm{cmc})$ of the anionic surfactants can be then associated to amorphous oxides reforming a layered material including the organic species or directly confined within an organo-LDH.

of the SDBS micelles that can be used as vector/carrier phases for the adsorption of PIC onto the layered materials. ${ }^{32}$ Since the UV-vis absorption technique provides quantitative data, the proper adsorption onto the organo-LDH could be confirmed by the large decrease in the absorption bands of a cationic dye. No residual PIC was detected in the supernatant after the washing procedure (stirring for 1 day) with water and a centrifugation step (5500 rpm for $15 \mathrm{~min}$ ). The absence of any release of PIC in the supernatants after a washing procedure (which was repeated twice) underlines the stability of the hybrid materials. Similar observations are found with the use of LDH oxides (Figure S8), suggesting that both routes based on organo-LDH or the oxides as possible host materials are possible for the obtention of stable hybrid organo-LDH including PIC.

By focusing on the investigation of the hybrid materials, TG analyses reveal weight losses related to decomposition of organics within the temperature range of $200-400{ }^{\circ} \mathrm{C}$. As PIC is associated with SBDS, the observed weight losses are rather hard to be assigned to only the thermal degradation of the dye that normally starts to be decomposed from $265^{\circ} \mathrm{C}$ and the decomposition lasts up to $330^{\circ} \mathrm{C}$ for a bulk PIC (Figure $2 \mathrm{~b}$ ). However, with the identification of vibrational modes of PIC, in particular, that at $1550 \mathrm{~cm}^{-1}$ attributed to the $\nu_{\mathrm{C}=\mathrm{N}}$ stretching mode of the cationic dye, ${ }^{4,15,28,32}$ FTIR spectroscopy confirms the proper adsorption of PIC onto the layered materials (Figure 2c) collected after washing with water and centrifugation processes. XRD represents a powerful tool to appreciate the changes in the structure of layered materials. In particular, the evolution of the angular position of the $00 \mathrm{l}$ reflection underlines the possible intercalation of the organic species, while the presence of additional patterns may indicate possible crystallization onto an external surface or a structural phase reorganization. Due to a memory effect property of $\mathrm{LDH}$, the use of the derivative amorphous oxides as a supporting phase drives to the reformation of a layered structure incorporating the anionic species within the internal space at about $30 \AA$. . The resulting hybrid materials display a slight increase in the repeating distance due to the intercalation of both PIC and SDBS. Similarly, the combination or association of SDBS micelles with PIC and an organo-LDH prepared with SDBS leads to a hybrid material showing a repeating distance slightly larger than that of the bulk SDBS$\mathrm{LDH}$, indicating the possible intercalation of PIC or reorganization of SDBS (Figure 2d). Within the interlayer space of the $\mathrm{LDH}$, the confinement of SDBS provides, due to the hydrophobic moieties of the anionic surfactant, a hydrophobic environment to the resulting hybrid materials, permitting the inclusion of additional molecules such as PIC to be intercalated in that way, as it could be observed in the case of organoclays. ${ }^{25}$ However, this slight increase in the interlayer could also result from adsorbed and intercalated water molecules as several works pointed out and thus hides the increase in interlayer space by the intercalation of the dye. The intercalation of relatively small molecules often drives to an absence of any increase in an interlayer space that remains identical to the initial starting materials. The latter was frequently observed using organoclays that did not show any changes in the internal structure after the adsorption/ intercalation of various large pharmaceuticals. ${ }^{18,19,22-24}$

Even if the TG data cannot be quantitatively used for a direct estimation of the adsorbed amount of PIC (due to the co-intercalation of both PIC and SDBS), UV-vis spectroscopy allows a possible determination of the adsorbed/intercalated amount of PIC which reaches a value of about $5.63 \times 10^{-4} \mathrm{~mol}$ $\mathrm{g}^{-1}$ of a layered material. By knowing the specific surface area of $\mathrm{LDH}$ (theoretically estimated at $520 \mathrm{~m}^{2} \mathrm{~g}^{-1}$ based on the knowledge of both the crystallographic parameters and the lattice of the $\mathrm{LDH}$ but several works stress out that the experimental specific surface area can be lower down to only $\left.40 \mathrm{~m}^{2} \mathrm{~g}^{-133-35}\right)$ and the apparent area of PIC $\left(0.68 \mathrm{~nm}^{2}\right)$, the adsorbed amount represents $45 \%$ of the total (both internal and external, with the external one representing only $2 \%$ of the whole surface) LDH surface, suggesting an intercalation of PIC. Nevertheless, the XRD patterns do not reveal any large difference in the repeating distance of the hybrid layered 

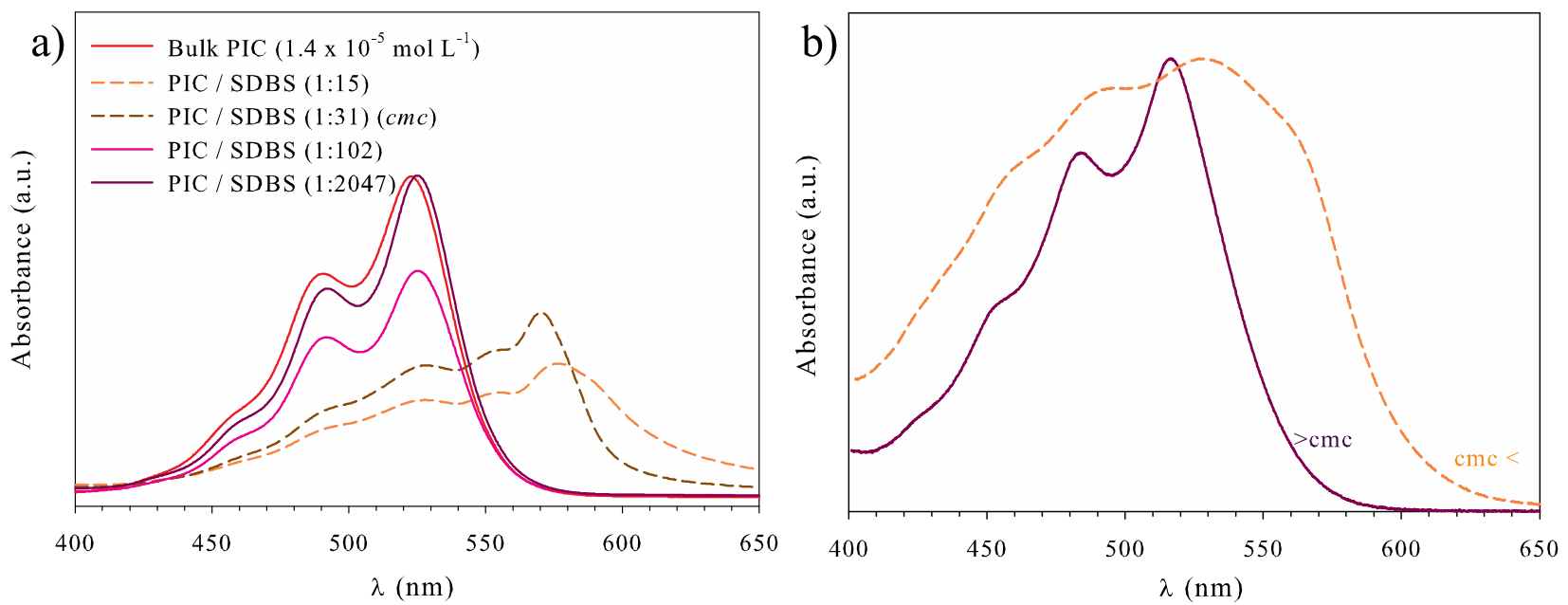

Figure 4. (a) UV-vis (liquid) spectra of bulk PIC solution and PIC-SDBS solutions with different molar ratios and concentrations of SDBS below and above the critical micelle concentration ( $\mathrm{cmc}$ ). (b) UV-vis (powder) spectra of the resulting hybrid material exhibiting the importance of the surfactant in solution (unimers or in spherical micelles).

materials after the co-adsorption of both PIC and SBDS even with possible intercalation of water molecules despite dehydration of the samples prior analyses. The hydrophobic environment and flexibility of the alkyl chains of the surfactants in the internal space of the hybrid materials can accommodate additional molecules without any large expansion of the distance of the inorganic galleries. The same trend was previously observed using organoclays as sorbent materials for the trapping of pollutants. The organoclays with-long-alkyl chain surfactants did not exhibit any changes in the interlayer space due to the flexibility of the hydrocarbon tails, making extra room in a material to sorb anionic pollutants. ${ }^{18,19,22-24}$

Thus, the combination of the whole data collected by TG, $\mathrm{UV}-$ vis, and infrared spectroscopies as well as XRD supports the intercalation of PIC into organo-LDHs (Figure 3) based on the strategy of the use of anionic surfactants (SDBS) as carrier/vector phases for the association with the layered materials.

3.3. Surfactant Dependence of the Photoluminescence Properties. The formation of dye aggregates depends on several parameters such as the presence of an inorganic surface and its geometry, $\mathrm{pH}$, and ionic strength, and the presence of other organic compounds (surfactants for instance). ${ }^{4,15,29,32}$ The aggregation of the dye occurs generally at high concentrations, and it is suppressed at high temperature while being also recognized to be affected by the presence of surfactants. ${ }^{32}$ Here, different experimental conditions such as SDBS concentrations (e.g., that for forming spherical micelles) as well as the nature of surfactants were investigated.

Above the cmc, anionic SDBS surfactants form spherical micelles which attract interests as vector or carrier phases for the intercalation of several organic compounds including PIC within layered materials. As expected and briefly discussed before, the presence of SDBS affects the UV-vis absorption profile with the observation of a red shift (Figures 2 and $4 a$ ). For bulk PIC in solution, three absorption bands are observed at 458,485 , and $521 \mathrm{~nm}$. The solubility of PIC dye is quite important; PIC dye (salt form) is easily solvated by water molecules and it is possible to consider that PIC dye in an aqueous solution does not pack with additional dye molecules. In contrast, in the presence of SDBS, the slight red shifts prove a change in the local environment of PIC due to their probable trapping within the SDBS spherical micelles, but without any formation of dimers in the core of the micelles for the studied concentration range. ${ }^{15,32}$ For the two investigated concentrations of SDBS below the $\mathrm{cmc}$, in addition to the monomer bands, a bathochromic shift is observed with the existence of new absorption bands at 560 and $570 \mathrm{~nm}$ which underlines the possible formation of $J$-aggregates after being associated to SDBS in unimers in the solution. The opposite electrical charges in addition to the lipophilic nature of both PIC and SDBS may favor a close association of the organic compounds below $\mathrm{cmc}$ that alter the energy levels of the PIC and cause the spectral shifts. $^{14}$

In addition to changing the PIC energy levels, surfactants play the role of carrier phases for the confinement of the cationic dyes within the internal structure of LDHs. The UVvis absorption spectra of the resulting hybrid materials obtained for SDBS in the $\mathrm{L}_{1}$ phase reveal the absence of any bathochromic shifts whatever the type of inorganic material used (i.e., organo-LDH or LDH derivative oxides). For the samples prepared above the $\mathrm{cmc}$, the large amount of SDBS prevents any possible aggregation of PIC leading to a molecular distribution of the whole PIC that thus show only monomer absorption bands. In contrast, as it can be observed with the SDBS-PIC solutions, the UV-vis spectra confirm a red shift of the absorption bands related to the PIC monomers (Figure $4 b$ ) as well as the existence of an absorption band at $570 \mathrm{~nm}$, suggesting the formation of $J$-aggregates in the hybrid materials. ${ }^{4,54}$ However, the absorption bands appear quite broad, underlining a poor control of the orientation of the dimers with multiple organization within the interlayer space. The confinement of the PIC dimers, assisted by the surfactants is likely to occur within the brushes formed by the SDBS alkyl chains. The flexibility of the hydrocarbon tails combined with their fast dynamics facilitate the insertion of PIC in the layered materials while limiting a fine orientation of the dyes. Thus, multiple orientations and configurations of the dimers are achieved without direct interaction between PIC and the inorganic $\mathrm{LDH}$ surface. Indeed, in contrast to previous research works with the use of large clay minerals, for instance, the direct interaction between the phyllosilicate sheets and PIC altered the energy level of PIC with the formation of $J$ aggregates lying parallel to the clay surface. ${ }^{4,5}$ In the latter case, 

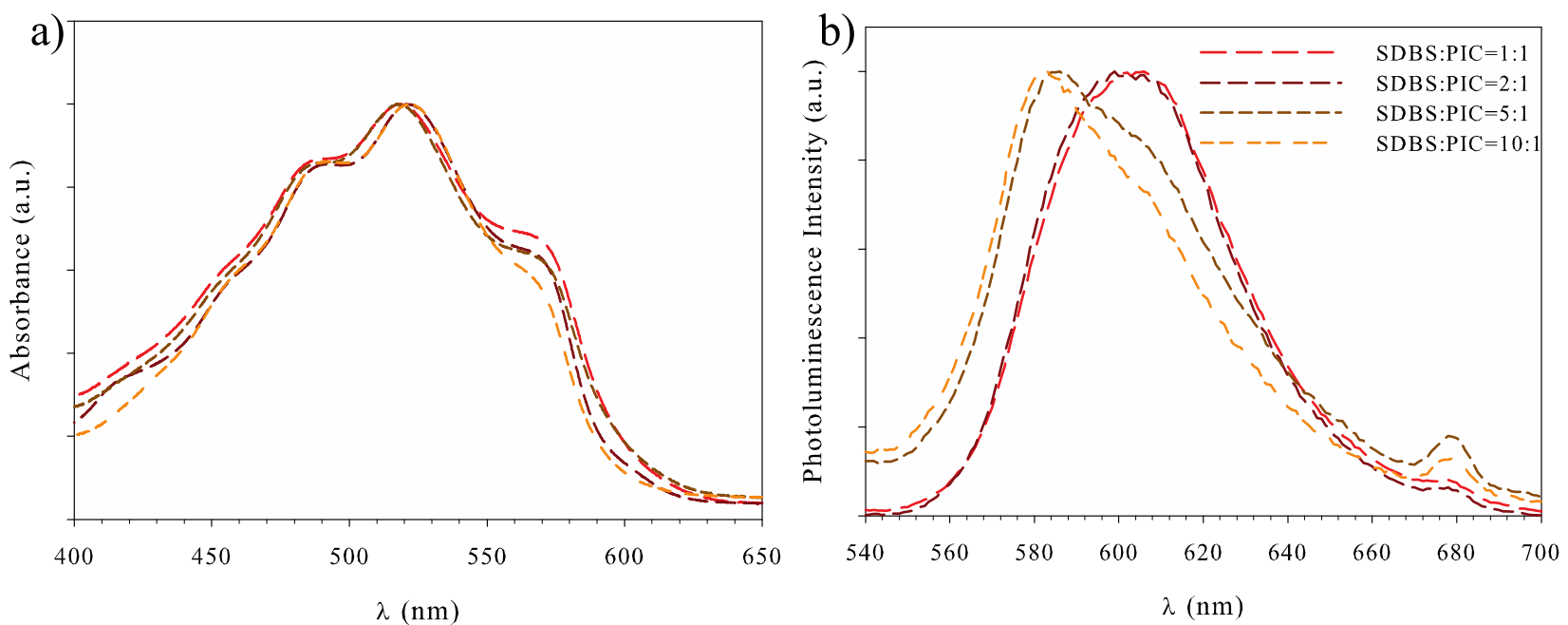

Figure 5. (a) UV-vis (powder) spectra of the hybrid layered materials prepared below the $\mathrm{cmc}$ for different PIC-SDBS molar ratios. (b) Photoluminescence spectra of the same hybrid layered materials after being irradiated at a wavelength of $450 \mathrm{~nm}$.
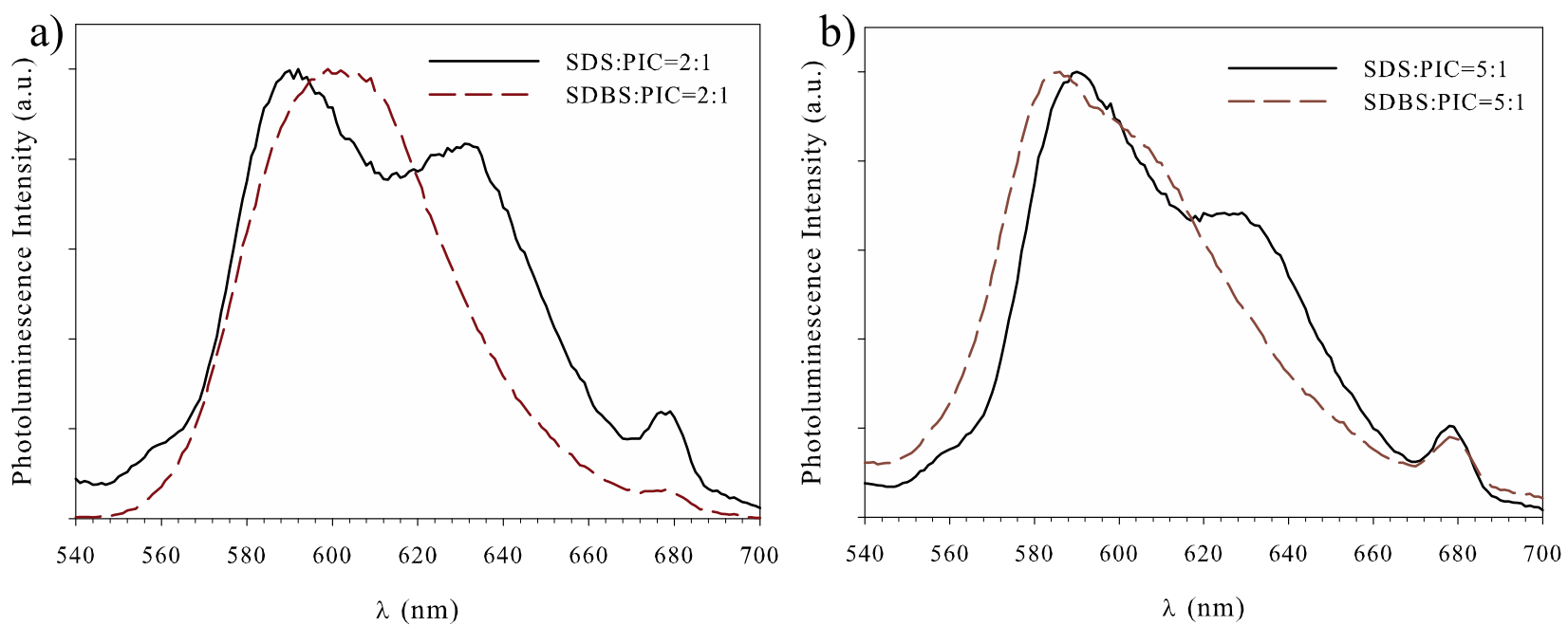

Figure 6. (a) Photoluminescence spectra of the layered materials prepared below the $\mathrm{cmc}$ with SDBS and SDS as carrier phases for the intercalation of a cationic dye (PIC) at different anionic surfactants: PIC ratio 2:1 (a) and 5:1 (b) (excitation wavelength of $450 \mathrm{~nm}$ ).

a sharp and intense absorption band was observed corresponding to resonant luminescence characteristics of $J$-aggregates.

Interestingly, whatever the routes taken for the preparation of the hybrid materials to include PIC through the use of organo- $\mathrm{LDH}$ or the derivative $\mathrm{LDH}$ oxides, the characterization of the solid-state UV-vis absorption properties of the hybrid materials display similar features (Figure 59). This underlines that the formation of an organo-LDH may occur first due to the excess of SDBS surfactants in solution (even below the $\mathrm{cmc}$ ) and then the PIC-SDBS organic complexes are intercalated in the interlayer space of the organo-layered material. In both routes, the hybrid materials show a lipophilic environment created by the alkyl-chain brushes, excluding any control of the PIC aggregates nor direct interaction between PIC and the LDH surface.

The solid-state UV-vis absorption spectra of different hybrid layered materials prepared for different SDBS:PIC ratios, while fixing the concentration of SDBS to $4.0 \times 10^{-4}$ mol L ${ }^{-1}$ (corresponding to the anion exchange capacity of $\mathrm{LDH}$ in the performed experimental protocol) below $\mathrm{cmc}$ displays the same features with the observation of both monomer bands and additional blue- and red-shifted bands related to the formation of $H$ - and $J$-aggregates, respectively (Figure 5a). However, it is interesting to note the decrease in the intensity of the J-band at about $580 \mathrm{~nm}$ with the increase in the SDBS concentration. This underlines the importance of the surfactant concentration that needs to be adjusted for the formation of the PIC dimers and explains the absence of absorption bands related to the aggregates (Figure 4) above $\mathrm{cmc}$. Below $\mathrm{cmc}$, the whole hybrid materials display broad Jbands, suggesting a short exciton delocalization length due to a high degree of static disorder in the J-aggregates. ${ }^{4,5,14}$ In contrast to nanoparticles and even clay minerals where the PIC molecules covered the surface through electrostatic interaction, forming the monomer molecules within $\pi$-interacting $J$ aggregate chains, the confinement of cationic dye occurring in a lipophilic environment of which SBDS density (more particularly the alkyl chains) controls the formation of the $J$ aggregates. However, the brush constituted by the alkyl chains does not permit a fine control of the order within the PIC aggregates, a scenario quite different from that of the $2 \mathrm{D}$ geometry surface may be provided by an $\mathrm{LDH}$ or a clay mineral surface whose lateral size was recognized to be a key 

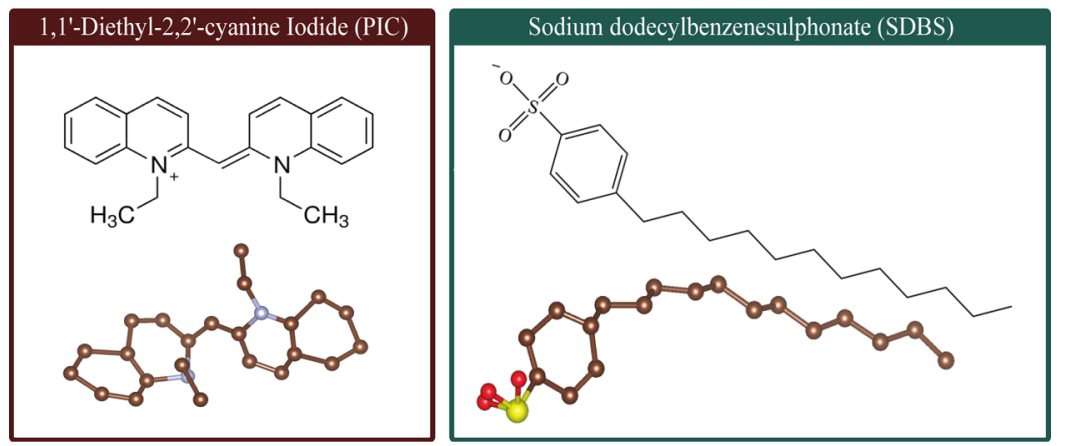

Figure 7. SDBS anionic surfactant used in this study as a chemical modifier for the preparation of the organo-LDH, as well as the carrier phase for the adsorption of PIC cationic dye within the layered materials.

parameter in the formation of the PIC aggregates, excluding any close interaction of the PIC to the inorganic surface. ${ }^{4,5}$

If the UV-vis absorption spectra of the hybrid materials do not show any drastic differences, the fluorescence spectra exhibit large differences with a dependence of the surfactant density. To note that the peak observed at $675 \mathrm{~nm}$ corresponds to the reflection at a half wavelength of the excitation light $(450 \mathrm{~nm})$, and thus, it is not related to any dye organization. At high loadings of the surfactant, the fluorescence intensity appears low (in the Figure $5 \mathrm{~b}$, the spectra were normalized to the maximum of fluorescence intensity with $\lambda_{\max }$ at about 585 $\mathrm{nm})$. Here again, the presence of the SBDS within the environment around the PIC molecule alters the fluorescence properties of a dye whose intensity maximum is expected at $580 \mathrm{~nm}$. The decrease in the surfactant at both ratios of $1: 1$ and 2:1 provokes a change in the fluorescence feedbacks with a shift to a high wavelength regime of the maximum of intensity $\lambda_{\text {max }}=605 \mathrm{~nm}$, due to the formation of the dye aggregates as well as the presence of a surfactant.

The close association of the surfactant with PIC modifies both UV-vis and photoluminescence properties of the resulting hybrid materials. The nature of the surfactant and its moieties (aromatic ring for instance) is recognized to control the type of aggregates. ${ }^{32}$ In particular, the inclusion of aromatic rings in the structure of surfactants leads to the formation of $J$-aggregates, while without aromatic rings but for the same length of the alkyl chains, the dyes are organized in $\mathrm{H}$-aggregate chains. Similar experiments were undertaken with sodium dodecyl sulfate (SDS) as vector/carrier phases for the intercalation of PIC in the organo-LDH materials. SDS shows the same alkyl chain length as SDBS without any benzyl group. As expected, the fluorescence spectra are sensitive to the nature of the surfactant and its density (Figure 6). Depending on the surfactant density that plays on the formation of the aggregates, an additional fluorescence peak or shoulder can be observed at about $640 \mathrm{~nm}$. However, as for SDBS, the broad fluorescence peaks appear indicating again a variety in the organization of the PIC aggregates without any fine orientation of the dye besides the use of 2D-layered materials as a template phase for the confinement and orientation of PIC. The incorporation of the surfactant enlarges the interlayer space while conferring to the hybrid materials a lipophilic environment. With the same alkyl-chain length, SDS forms a hydrophobic brush with a poor control of the additional organic species including PIC. Finally, the latter proves the importance of the structure of the surfactant, its density, and state in solution for the preparation of the hybrid layered materials based on the dye and their photoluminescence properties.

\section{CONCLUSIONS}

Layered materials have been commonly used to confine and orientate dyes, leading to the formation of singular assemblage showing photographic spectral sensitization (the case of $J$ aggregates). The formation of these dye aggregates within the internal structure of the layered materials can be obtained at much lower density than in bulk systems. Similarly, the use of surfactants and their structures play an important role in the control of the dye aggregates. In this study, we associate the action of both surfactants and layered materials for the confinement of a cationic dye. However, in contrast to previous studies aiming at the co-intercalation of both anionic dye and surfactants, we combine a cationic dye (PIC) and anionic surfactants acting as the carrier phase for the intercalation of the PIC within the interlayer space of LDH. Confined in $\mathrm{LDH}$, the formation of the aggregates mainly depends on the surfactant state in solution (spherical micelle or monomers), its density, and its structure (aromatic moiety).

Due to their confinement within the lipophilic media conferred by the presence of the flexible alkyl chains, $J$ aggregates display a high degree of static disorder leading to poor UV-vis absorption and fluorescence properties. However, this study tackles a novel route for the intercalation of a cation in LDH assisted by anionic surfactants. Further efforts will be devoted in the understanding on the lateral size, composition of the $\mathrm{LDH}$, and the structure and density of the anionic surfactants used in regard to the anion exchange capacity of the layered materials.

\section{MATERIALS AND METHODS}

4.1. Materials. Magnesium chloride hexahydrate $\left(\mathrm{MgCl}_{2}\right.$. $6 \mathrm{H}_{2} \mathrm{O},>99.0 \%$, Kanto), aluminum(III) chloride ( $\mathrm{AlCl}_{3}$, $>98.0 \%$, Junsei), urea $\left(\mathrm{NH}_{2} \mathrm{CONH}_{2},>99.0 \%\right.$, Kanto), SDBS $\left(\mathrm{C}_{18} \mathrm{H}_{29} \mathrm{NaO}_{3} \mathrm{~S},>95.0 \%\right.$, TCI $)$, and 1,1'-diethyl-2,2'-cyanine iodide (PIC) as a cationic dye $\left(\mathrm{C}_{23} \mathrm{H}_{23} \mathrm{IN}_{2}>98.0 \%\right.$, Tokyo Kasei Kogyo) were used without further purification (Figure 7).

4.2. Synthesis of $\mathrm{CO}_{3}-\mathrm{Mg} / \mathrm{Al} \mathrm{LDH}$. $\mathrm{LDH}$ was prepared as reported. ${ }^{27} \mathrm{MgCl}_{2} \cdot 6 \mathrm{H}_{2} \mathrm{O}$ (6.78 g, $33.3 \mathrm{mmol}$ ), $\mathrm{AlCl}_{3}$ (2.22 g, $16.6 \mathrm{mmol})$, and urea $(10.0 \mathrm{~g}, 167 \mathrm{mmol})$ were dissolved in pure water and sealed in an autoclave. The autoclave was heated at $120{ }^{\circ} \mathrm{C}$ for $36 \mathrm{~h}$. The product was separated by filtration and washed with water. After a vacuum-drying 
process performed at room temperature for 1 day, $\mathrm{CO}_{3}-\mathrm{Mg}$ / $\mathrm{Al} \mathrm{LDH}$ was obtained.

4.3. Synthesis of SDBS-LDH and the Hybrid Layered Materials Including PIC. $\mathrm{CO}_{3}-\mathrm{Mg} / \mathrm{Al} \mathrm{LDH}$ was introduced in a crucible and heated at $500{ }^{\circ} \mathrm{C}$ for $20 \mathrm{~h}$ in order to remove $\mathrm{CO}_{3}{ }^{2-}$ anions (c-LDH). In the round-bottom flask, c-LDH $(100 \mathrm{mg})$ and SDBS solution $\left(5.00 \mathrm{~mL}, 0.200 \mathrm{~mol} \mathrm{~L}^{-1}\right)$ were added and stirred at room temperature for 1 day under a $\mathrm{N}_{2}$ atmosphere. The white precipitate was separated by centrifugation and washed with water (SDBS-LDH). SDBS$\mathrm{LDH}$ was dispersed and exfoliated in deionized water.

In a vial, c-LDH was dispersed in deionized water under a $\mathrm{N}_{2}$ atmosphere. To this dispersion, a solution of SDBS and PIC was added, changing the molar ratio of SDBS to PIC to $1: 1,2: 1,3: 1,4: 1,5: 1$, and $10: 1$ and using a concentration of $4.0 \times 10^{-4} \mathrm{~mol} \mathrm{~L}^{-1}$ (below $\mathrm{cmc}$ ). In addition, solutions at a large concentration of SDBS (above $\mathrm{cmc}$ ) including PIC (at a concentration of $7.05 \mathrm{~mol} \mathrm{~L}^{-1}$ ) were used to probe the intercalation of PIC within the internal structure of the layered materials. After stirring at room temperature for 1 day and a centrifugation process, the hybrid materials were collected and washed two times with deionized water. Similar experiments were undertaken with the use of SDBS-LDH dispersion.

4.4. Analyses. XRD patterns were obtained with a Rigaku Rint-2500 diffractometer under the following conditions: 40 $\mathrm{kV}, 30 \mathrm{~mA}$, and $\mathrm{Cu} \mathrm{K} \alpha$ radiation. The powder samples were recorded with a step of $0.04^{\circ}(2 \theta)$ in the range from 1.5 to $80^{\circ}$ with a scanning rate of $0.5^{\circ} / \mathrm{min}$. FTIR spectra were obtained with an FT/IR-460 Plus spectrometer (JASCO) using the $\mathrm{KBr}$ disk method. The morphology of the LDH sheets was investigated using scanning electron microscopy (SEM, JEOL IT-100 LA). TG analyses were determined using a TG8120 thermobalance (Rigaku) with a heating rate of $10{ }^{\circ} \mathrm{C} / \mathrm{min}$ under air flow. The UV-vis absorption spectra of the solutions were collected on a Shimadzu UV-3100PC spectrophotometer with the measurement range between 190 and $320 \mathrm{~nm}$. UV analyses on solid samples (powder form) were performed on a Jasco V-750 spectrophotometer with the wavelength range between 190 and $900 \mathrm{~nm}$ and the use of $\mathrm{BaSO}_{4}$ as a reference. The solution and solid-state fluorescence spectra were recorded on a Jasco V-750 spectrophotometer with the use of an excitation wavelength at $450 \mathrm{~nm}$.

\section{ASSOCIATED CONTENT}

\section{SI Supporting Information}

The Supporting Information is available free of charge at https://pubs.acs.org/doi/10.1021/acsomega.1c02724.

Synthesis and characterization of the $\mathrm{Mg}-\mathrm{Al} \mathrm{LDHs}$ obtained by the urea method: XRD, FTIR, and SEM; calcination and characterization of the oxidized $\mathrm{LDH}$; collapse of the layered structure was confirmed by XRD and the oxidation by FTIR; XRD and FTIR characterization of the hybrid layered materials obtained with SDBS; and UV-visible characterizations of the hybrid materials including PIC based on $\mathrm{LDH}$ oxides (PDF)

\section{AUTHOR INFORMATION}

\section{Corresponding Author}

Régis Guégan - Global Center for Science and Engineering, Faculty of Science and Engineering, Waseda University, Tokyo 169-8050, Japan; 이이.org/0000-0002-81526826; Email: regis.guegan@aoni.waseda.jp

\section{Authors}

Rina Sato - Department of Applied Chemistry, Waseda University, Tokyo 169-8050, Japan

Shingo Machida - Department of Applied Chemistry, Waseda University, Tokyo 169-8050, Japan; Tokyo University of Science, Musashino 162-8601 Tokyo, Japan; 으이.org/ 0000-0002-7574-3496

Minoru Sohmiya - Tokyo University of Science, Musashino 162-8601 Tokyo, Japan; Seikei University, Musashino 1808633 Tokyo, Japan

Yoshiyuki Sugahara - Department of Applied Chemistry, Waseda University, Tokyo 169-8050, Japan; 이이.org/ 0000-0003-2973-8052

Complete contact information is available at:

https://pubs.acs.org/10.1021/acsomega.1c02724

\section{Notes}

The authors declare no competing financial interest.

\section{REFERENCES}

(1) Belbel, A.; Kharroubi, M.; Janot, J.-M.; Abdessamad, M.; Haouzi, A.; Lefkaier, I. K.; Balme, S. Preparation and characterization of homoionic montmorillonite modified with ionic liquid: Application in dye adsorption. Colloids Surf., A 2018, 558, 219-227.

(2) Tangaraj, V.; Janot, J.-M.; Jaber, M.; Bechelany, M.; Balme, S. Adsorption and photophysical properties of fluorescent dyes over montmorillonite and saponite modified by surfactant. Chemosphere 2017, 184, 1355-1361.

(3) Chakraborty, C.; Dana, K.; Malik, S. Intercalation of Perylenediimide Dye into LDH Clays: Enhancement of Photostability. J. Phys. Chem. C 2011, 115, 1996-2004.

(4) Miyamoto, N.; Kawai, R.; Kuroda, K.; Ogawa, M. Adsorption and aggregation of a cationic cyanine dye on layered clay minerals. Appl. Clay Sci. 2000, 16, 161-170.

(5) Yamaguchi, T.; Oh, J.-M.; Ogawa, M. Photofunctions of DyeClay Hybrids: Recent Developments. In Dyes and Photoactive Molecules in Microporous Systems; Martínez-Martínez, V., López Arbeloa, F., Eds.; Springer International Publishing: Cham, 2020; pp 251-320.

(6) Yan, D.; Lu, J.; Ma, J.; Wei, M.; Qin, S.; Chen, L.; Evans, D. G.; Duan, $\mathrm{X}$. Thin film of coumarin-3-carboxylate and surfactant cointercalated layered double hydroxide with polarized photoluminescence: a joint experimental and molecular dynamics study. J. Mater. Chem. 2010, 20, 5016-5024.

(7) Yan, D.; Lu, J.; Wei, M.; Evans, D. G.; Duan, X. Recent advances in photofunctional guest/layered double hydroxide host composite systems and their applications: experimental and theoretical perspectives. J. Mater. Chem. 2011, 21, 13128-13139.

(8) Yan, D.; Lu, J.; Wei, M.; Ma, J.; Evans, D. G.; Duan, X. A combined study based on experiment and molecular dynamics: perylene tetracarboxylate intercalated in a layered double hydroxide matrix. Phys. Chem. Chem. Phys. 2009, 11, 9200-9209.

(9) Imwiset, K.; Ogawa, M. Characteristics of flexible supramolecular assembly of dioleyldimethylammonium ion confined in a two dimensional nanospace studied by the host-guest reactions. Colloids Surf., A 2020, 605, 125352.

(10) Imwiset, K. J.; Ogawa, M. Highly Luminescent InorganicOrganic Hybrids with Molecularly Dispersed Perylene. Inorg. Chem. 2021, 60, 9563.

(11) Sanati, S.; Rezvani, Z. Co-intercalation of Acid Red-27/sodium dodecyl sulfate in a Ce-containing Ni-Al-layered double hydroxide matrix and characterization of its luminescent properties. J. Mol. Liq. 2018, 249, 318-325.

(12) Sun, Z.; Jin, L.; Shi, W.; Wei, M.; Evans, D. G.; Duan, X. Controllable Photoluminescence Properties of an Anion-DyeIntercalated Layered Double Hydroxide by Adjusting the Confined Environment. Langmuir 2011, 27, 7113-7120. 
(13) Yan, D.; Lu, J.; Ma, J.; Wei, M.; Evans, D. G.; Duan, X. Benzocarbazole anions intercalated layered double hydroxide and its tunable fluorescence. Phys. Chem. Chem. Phys. 2010, 12, 1508515092 .

(14) Yefimova, S. L.; Grygorova, G. V.; Klochkov, V. K.; Borovoy, I. A.; Sorokin, A. V.; Malyukin, Y. V. Molecular Arrangement in Cyanine Dye J-Aggregates Formed on $\mathrm{CeO} 2$ Nanoparticles. J. Phys. Chem. C 2018, 122, 20996-21003.

(15) Lanzafame, J. M.; Muenter, A. A.; Brumbaugh, D. V. The effect of J-aggregate size on photoinduced charge transfer processes for dyesensitized silver halides. Chem. Phys. 1996, 210, 79-89.

(16) Costa, F. R.; Leuteritz, A.; Wagenknecht, U.; Jehnichen, D.; Häußler, L.; Heinrich, G. Intercalation of $\mathrm{Mg}-\mathrm{Al}$ layered double hydroxide by anionic surfactants: Preparation and characterization. Appl. Clay Sci. 2008, 38, 153-164.

(17) Costantino, U.; Coletti, N.; Nocchetti, M.; Aloisi, G. G.; Elisei, F.; Latterini, L. Surface Uptake and Intercalation of Fluorescein Anions into $\mathrm{Zn}-\mathrm{Al}-\mathrm{Hydrotalcite}$. Photophysical Characterization of Materials Obtained. Langmuir 2000, 16, 10351-10358.

(18) De Oliveira, T.; Boussafir, M.; Fougère, L.; Destandau, E.; Sugahara, Y.; Guégan, R. Use of a clay mineral and its nonionic and cationic organoclay derivatives for the removal of pharmaceuticals from rural wastewater effluents. Chemosphere 2020, 259, 127480.

(19) De Oliveira, T.; Fernandez, E.; Fougère, L.; Destandau, E.; Boussafir, M.; Sohmiya, M.; Sugahara, Y.; Guégan, R. Competitive Association of Antibiotics with a Clay Mineral and Organoclay Derivatives as a Control of Their Lifetimes in the Environment. ACS Omega 2018, 3, 15332-15342.

(20) De Oliveira, T.; Guégan, R. Coupled Organoclay/Micelle Action for the Adsorption of Diclofenac. Environ. Sci. Technol. 2016, 50, 10209-10215.

(21) De Oliveira, T.; Guégan, R.; Thiebault, T.; Milbeau, C. L.; Muller, F.; Teixeira, V.; Giovanela, M.; Boussafir, M. Adsorption of diclofenac onto organoclays: Effects of surfactant and environmental ( $\mathrm{pH}$ and temperature) conditions. J. Hazard. Mater. 2017, 323, 558566.

(22) Guégan, R. Organoclay applications and limits in the environment. C. $R$. Chim. 2019, 22, 132-141.

(23) Guégan, R.; De Oliveira, T.; Le Gleuher, J.; Sugahara, Y. Tuning down the environmental interests of organoclays for emerging pollutants: Pharmaceuticals in presence of electrolytes. Chemosphere 2020, 239, 124730 .

(24) Guégan, R.; Le Forestier, L. Performance evaluation of organoclays for the amoxicillin retention in a dynamic context. Chem. Eng. J. 2021, 406, 126859.

(25) Guégan, R.; Veron, E.; Le Forestier, L.; Ogawa, M.; Cadars, S. Structure and Dynamics of Nonionic Surfactant Aggregates in Layered Materials. Langmuir 2017, 33, 9759-9771.

(26) He, Y.; Wang, R.; Jiao, T.; Yan, X.; Wang, M.; Zhang, L.; Bai, Z.; Zhang, Q.; Peng, Q. Facile Preparation of Self-Assembled Layered Double Hydroxide-Based Composite Dye Films As New Chemical Gas Sensors. ACS Sustainable Chem. Eng. 2019, 7, 10888-10899.

(27) Ogawa, M.; Kaiho, H. Homogeneous Precipitation of Uniform Hydrotalcite Particles. Langmuir 2002, 18, 4240-4242.

(28) Matejdes, M.; Himeno, D.; Suzuki, Y.; Kawamata, J. The effect of the negative charge density on switchable properties of pseudoisocyanine dye. Appl. Clay Sci. 2017, 144, 54-59.

(29) Khairutdinov, R. F.; Serpone, N. Photophysics of Cyanine Dyes: Subnanosecond Relaxation Dynamics in Monomers, Dimers, and $\mathrm{H}-$ and J-Aggregates in Solution. J. Phys. Chem. B 1997, 101, $2602-2610$

(30) Szadkowski, B.; Marzec, A.; Rogowski, J.; Maniukiewicz, W.; Zaborski, M. Insight into the formation mechanism of azo dye-based hybrid colorant: Physico-chemical properties and potential applications. Dyes Pigm. 2019, 167, 236-244.

(31) Salleres, S.; López Arbeloa, F.; Martínez, V.; Corcóstegui, C.; López Arbeloa, I. Effect of surfactant C12TMA molecules on the selfassociation of R6G dye in thin films of laponite clay. Mater. Chem. Phys. 2009, 116, 550-556.
(32) McHedlov-Petrossyan, N. O.; Shapovalov, S. A.; Koval, V. L.; Shakhverdov, T. A.; Bochkaryov, Y. A. the surfactant-induced formation of $\mathrm{J}$ - and $\mathrm{H}$-aggregates in aqueous pseudoisocyanine solutions. Dyes Pigm. 1992, 19, 33-40.

(33) You, Y.; Zhao, H.; Vance, G. F. Surfactant-enhanced adsorption of organic compounds by layered double hydroxides. Colloids Surf., A 2002, 205, 161-172.

(34) Zhang, P.; Wang, T.; Qian, G.; Frost, R. L. Organo-LDH synthesized via tricalcium aluminate hydration in the present of $\mathrm{Na}$ dodecylbenzenesulfate aqueous solution and subsequent investigated by near-infrared and mid-infrared. Spectrochim. Acta, Part A 2014, $125,195-200$.

(35) Chen, C.; Wangriya, A.; Buffet, J.-C.; O’Hare, D. Tuneable ultra high specific surface area $\mathrm{Mg} / \mathrm{Al}-\mathrm{CO} 3$ layered double hydroxides. Dalton Trans. 2015, 44, 16392-16398. 